\title{
ATENÇÃO NUTRICIONAL ÀS GESTANTES DE BAIXO RISCO: CONTRIBUIÇÕES PARA AS POLÍTICAS PÚBLICAS
}

\section{Leticia Alves Soares}

Nutricionista Residente. Programa de Residência Multiprofissional em Saúde da Família. Universidade Federal de Alfenas (UNIFAL-MG), Brasil.

\section{Daniela Braga Lima}

Nutricionista. Docente Adjunto na Faculdade de Nutrição e no Programa de Residência Multiprofissional em Saúde da Família. Universidade Federal de Alfenas (UNIFAL-MG), Brasil.
RESUMO: Trata-se de estudo transversal com objetivo de avaliar a atenção nutricional de gestantes de baixo risco, cadastradas em Estratégias de Saúde da Família. A coleta de dados foi realizada por meio de um questionário estruturado com informações socioeconômicas, obstétricas, estilo de vida e nutricionais. O estado nutricional foi analisado pelo Índice de Massa Corporal pré-gestacional/gestacional e ganho ponderal. Para avaliar o consumo alimentar foi aplicado o Recordatório Alimentar de 24 horas. Das 70 gestantes estudadas verificou-se que $22,9 \%$ eram de baixo peso e $40,0 \%$ estavam com excesso de peso. Os dados evidenciaram a associação significativa entre o Índice de Massa Corporal pré-gestacional e o estado nutricional $(\mathrm{p}<0,000)$. Houve prevalência de ganho ponderal insuficiente, e a qualidade da alimentação da população avaliada mostrou-se aquém das recomendações preconizadas. Faz-se necessária maior atenção à população estudada, para melhoria da qualidade da atenção nutricional, incluindo o cuidado multiprofissional, na rede primária de saúde.

PALAVRAS-CHAVE: Gravidez; Avaliação nutricional; Cuidado prénatal; Atenção primária à saúde.

\section{NUTRITIONAL CARE FOR LOW-RISK PREGNANT WOMEN: SOME CONTRIBUTIONS FOR PUBLIC POLICIES}

\begin{abstract}
Current transversal study evaluated the nutritional care of low-risk pregnant women enrolled in Family Health Strategies. Data were collected through a questionnaire on socio-economical, obstetrical, lifestyle and nutritional information. Nutritional status was assessed by pre-pregnancy/pregnancy Body Mass Index (BMI) and weight gain and food consumption was evaluated by a 24-h Food Record. Further, $22.9 \%$ of the 70 pregnant women had low weight and $40.0 \%$ were overweight. Data revealed a significant association between pre-pregnancy BMI and nutritional state $(\mathrm{p}<0.000)$. Insufficient weight gain was prevalent and food quality of the evaluated population was below that recommended. Greater care to the population studied is required for better nutritional quality by multiprofessional care in first aid health units.
\end{abstract}

KEY WORDS: Pregnancy; Nutrition Assessment; Prenatal Care; Primary Health Care. 


\section{INTRODUÇÃO}

A gestação é caracterizada por um período de intensas alterações fisiológicas, metabólicas e endócrinas, constituindo-se, assim, em momento de maior vulnerabilidade, principalmente, quanto ao estado nutricional inicial, ganho de peso gestacional e consumo alimentar que têm implicações diretas na saúde maternoinfantil ${ }^{1-3}$.

A alimentação adequada é de suma importância durante a gestação, pois a demanda de nutrientes e energia tende a aumentar devido às transformações fisiológicas. Em contrapartida, os desequilíbrios na alimentação de gestantes têm sido associados ao nascimento de bebês pré-termos e com comprometimento do desenvolvimento e crescimento do feto ${ }^{4-5}$.

A avaliação dietética da gestante por meio de inquéritos alimentares é importante, pois possibilita detectar problemas nutricionais específicos já existentes, que podem ser prejudiciais no decorrer da gestação. Para tanto, é fundamental o cuidado e atenção nutricional à saúde da mulher durante a gravidez, à saúde do concepto, ao bem-estar materno para possibilitar a nutrição do recém-nascido e à proteção contra o desenvolvimento de doenças crônicas durante a vida adulta ${ }^{6}$.

Assim, o estado nutricional materno, com o monitoramento também do ganho do peso, antes e durante a gestação, é um forte determinante para o desfecho da gestação e a saúde da mãe e do recémnascido ${ }^{7}$.

As variações no ganho de peso durante a gestação podem ser influenciadas tanto por fatores nutricionais como sociodemográficos, obstétricos e comportamentais. ${ }^{6}$ Ainda, esse acompanhamento do ganho ponderal permite identificar situações de riscos gestacionais e auxilia no estabelecimento de intervenções nutricionais adequadas ${ }^{8}$.

Tendo em vista a importância da atenção nutricional no pré-natal, faz-se necessária a adequação na alimentação da gestante, visto que o estado nutricional preservado pode contribuir positivamente para os desfechos gestacionais?.

Por tais motivos, cabe afirmar, que o estado nutricional pré-gestacional e o ganho ponderal têm impacto positivo na saúde materno-fetal e no pósparto; este estudo teve como objetivo avaliar a atenção nutricional de gestantes de baixo risco cadastradas em Estratégias de Saúde da Família (ESF).

\section{METODOLOGIA}

Trata-se de estudo transversal realizado com 70 gestantes de um total de 82 identificadas no cadastro do Sistema de Monitoramento e Avaliação do Pré-natal, Parto, Puerpério e Criança - ESF do município de Alfenas/MG, no período de novembro de 2016 a junho de 2017. Foram consideradas elegíveis as gestantes que participavam regularmente do pré-natal e aceitaram, voluntariamente, participar do estudo. Foram excluídas as gestantes de alto risco (hipertensas, diabéticas, gemelares), adolescentes (período que compreende a idade entre 10 aos 19 anos), conforme critério adotado pela Organização Mundial de Saúde ${ }^{10} \mathrm{e}$ as que abandonaram o pré-natal.

A coleta de dados foi realizada por meio de um questionário estruturado com informações socioeconômicas, obstétricas, estilo de vida e nutricionais. As medidas antropométricas de peso e altura foram aferidas com base nos critérios de padronização do Ministério da Saúde ${ }^{11}$.

Para classificar o estado nutricional prégestacional foram utilizados os pontos de corte propostos pelo Institute Of Medicine ${ }^{12}$ e adotados pelo Ministério da Saúde ${ }^{11}$. De acordo com tal classificação, mulheres com Índice de Massa Corporal (IMC) pré-gestacional menor de $18,5 \mathrm{~kg} / \mathrm{m}^{2}$ eram de baixo peso; eutróficas, com IMC de 18,5 a $24,9 \mathrm{~kg} / \mathrm{m}^{2}$; sobrepeso, com IMC entre 25 a $29,9 \mathrm{~kg} / \mathrm{m}^{2}$; e obesas, com IMC acima de $30 \mathrm{~kg} / \mathrm{m}^{2}$. Já o estado nutricional das gestantes no momento da coleta dos dados foi avaliado considerando os níveis críticos de IMC para idade gestacional, conforme recomendações do Ministério da Saúde ${ }^{11}$.

Para classificar a adequação do ganho de peso gestacional foram utilizados os critérios do Institute of Medicine $^{12}$, que estabelecem limites para o ganho de peso gestacional de acordo com o estado nutricional inicial da gestante. O ganho ponderal foi classificado nas seguintes categorias: ganho de peso insuficiente, adequado ou excessivo. 
Para mensurar o consumo alimentar aplicouse o Recordatório Alimentar de 24 horas. O cálculo do consumo alimentar foi realizado por meio do programa Dietbox $^{\circledR}$ (TIME DIETBOX, 2016). Para avaliar a adequação da ingestão utilizaram-se as Dietary Reference Intakes (DRI) como valores de referência ${ }^{13}$.

Os resultados foram analisados por meio do programa Statistical Package for Social Sciences (SPSS) 22.0. Na análise descritiva foram calculadas as frequências absolutas (n) e relativas (\%). Para as variáveis quantitativas foram obtidos os valores de média e desvios-padrão. Para comparação entre proporções foi usado o teste do quiquadrado e o teste exato de Fisher quando necessário. Foi utilizado o teste ANOVA para a comparação das médias entre os grupos nas variáveis com distribuição normal, e o teste de Kruskal-Wallis para as variáveis de distribuição não normal. Para identificação da significância dos grupos foi aplicado o teste de Tukey. Para todos os testes utilizados foi adotado o nível de significância de 5\%.

As participantes deste estudo foram esclarecidas durante sua triagem pelo pesquisador responsável, sobre o objetivo da pesquisa, bem como sobre sua participação voluntária e confiabilidade dos dados e só participaram após assinarem o Termo de Consentimento Livre e Esclarecido (TCLE). O estudo foi aprovado pelo Comitê de Ética em Pesquisa da Universidade Federal de Alfenas (Unifal/MG) (número do parecer: 1.817.738).

\section{RESULTADO}

O perfil das gestantes atendidas nas Estratégias de Saúde da Família evidenciou que 77,1\% encontravamse na faixa etária entre 20 a 30 anos, e 38,6\% estavam no segundo trimestre de gestação no momento da coleta de dados. No tocante às características socioeconômica $\mathrm{e}$ estilo de vida, verificou-se a maior prevalência de união estável (62,9\%), não exerciam atividade remunerada $(70,0 \%)$ e renda familiar inferior ou igual a um salário (55,7\%). Quanto à escolaridade, 50,0\% de gestantes relataram menor de oito anos. Ainda, o tabagismo e consumo de álcool foram relatados por 11,4 e 8,6\% das entrevistadas, respectivamente (Tabela 1).
Tabela 1. Características socioeconômicas, obstétricas e estilo de vida das gestantes atendidas em Estratégias de Saúde da Família, Alfenas-MG, 2017

\begin{tabular}{|c|c|c|}
\hline Características & $\mathbf{n}$ & $\%$ \\
\hline \multicolumn{3}{|l|}{ Faixa etária } \\
\hline $20-30$ anos & 54 & 77,1 \\
\hline$>30$ anos & 16 & 22,9 \\
\hline \multicolumn{3}{|l|}{$\begin{array}{l}\text { Idade gestacional } \\
\text { atual }\end{array}$} \\
\hline $1^{\circ}$ trimestre & 23 & 32,9 \\
\hline $2^{\circ}$ trimestre & 27 & 38,6 \\
\hline $3^{\circ}$ trimestre & 20 & 28,6 \\
\hline \multicolumn{3}{|l|}{ Situação marital } \\
\hline Sem companheiro & 26 & 37,1 \\
\hline Com companheiro & 44 & 62,9 \\
\hline \multicolumn{3}{|l|}{ Escolaridade } \\
\hline$<8$ anos & 35 & 50,0 \\
\hline$\geq 8$ anos & 35 & 50,0 \\
\hline \multicolumn{3}{|l|}{ Renda familiar* } \\
\hline$\leq 1$ salário & 39 & 55,7 \\
\hline$>1$ salário & 31 & 44,3 \\
\hline \multicolumn{3}{|l|}{ Ocupação } \\
\hline Não remunerada & 49 & 70,0 \\
\hline Remunerada & 21 & 30,0 \\
\hline \multicolumn{3}{|l|}{$\begin{array}{l}\text { Consumo de } \\
\text { álcool na gestação }\end{array}$} \\
\hline Sim & 6 & 8,6 \\
\hline Não & 64 & 91,4 \\
\hline \multicolumn{3}{|l|}{$\begin{array}{l}\text { Tabagismo na } \\
\text { gestação }\end{array}$} \\
\hline Sim & 8 & 11,4 \\
\hline Não & 62 & 88,6 \\
\hline
\end{tabular}

Nota:*salário mínimo R\$937,00 


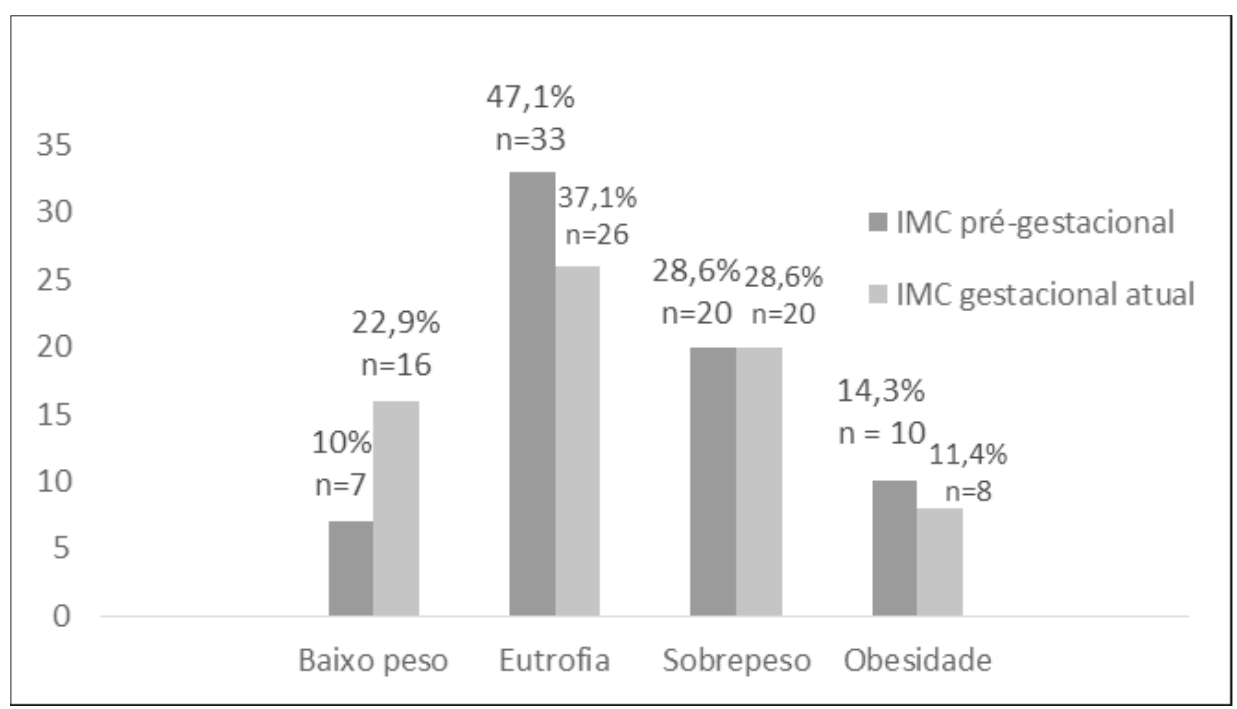

Figura 1. Estado Nutricional pré- gestacional e gestacional atual de gestantes atendidas em Estratégias de Saúde da Família, Alfenas-MG, 2017

Na Figura 1 é mostrado que 22,9\% e 40,0\% das gestantes pesquisadas encontravam-se com baixo e excesso de peso para idade gestacional, respectivamente. No entanto, observou-se que o número de mulheres com baixo peso dobrou durante o período gestacional quando comparado com o estado nutricional inicial (aumento de $12,9 \%$ ), por outro lado, os percentuais de gestantes que apresentaram sobrepeso e obesidade se mantiveram praticamente estáveis.

O ganho ponderal materno insuficiente foi observado em $52,9 \%$ das gestantes, enquanto o ganho ponderal excessivo esteve presente em $14,2 \%$ das mulheres. Metade das gestantes que apresentou ganho de peso excessivo encontrava-se no segundo trimestre gestacional. Observou-se associação estatística (Valor $p$ $=0,022)$ apenas entre a situação marital e o ganho de peso semanal. Na análise em relação ao ganho ponderal recomendado para a idade gestacional, verificou-se que o ganho ponderal foi adequado em proporção maior nas gestantes com eutrofia e sobrepeso, no entanto, não houve associação do ganho ponderal materno com o estado nutricional (Tabela 2).

Tabela 2. Características socioeconômicas, obstétricas e nutricionais segundo ganho ponderal total de gestantes atendidas em Estratégias de Saúde da Família, Alfenas-MG, 2017

(Continua)

\begin{tabular}{|c|c|c|c|c|c|c|c|}
\hline \multirow{3}{*}{ Variáveis } & \multicolumn{6}{|c|}{ Ganho ponderal total } & \multirow{3}{*}{ Valor $\mathrm{p}$} \\
\hline & \multicolumn{2}{|c|}{$\begin{array}{l}\text { Insuficiente } \\
\quad(n=37)\end{array}$} & \multicolumn{2}{|c|}{$\begin{array}{l}\text { Adequado } \\
(\mathrm{n}=23)\end{array}$} & \multicolumn{2}{|c|}{$\begin{array}{c}\text { Excessivo } \\
(n=10)\end{array}$} & \\
\hline & $\mathrm{n}$ & $\%$ & $\mathrm{n}$ & $\%$ & $\mathrm{n}$ & $\%$ & \\
\hline Faixa etária & & & & & & & 0,845 \\
\hline $20-30$ anos & 29 & 78,4 & 18 & 78,3 & 7 & 70,0 & \\
\hline$>30$ anos & 8 & 21,6 & 5 & 21,7 & 3 & 30,0 & \\
\hline \multicolumn{8}{|c|}{ Idade gestacional atual } \\
\hline $1^{\circ}$ trimestre & 15 & 40,5 & 5 & 21,7 & 3 & 30,0 & 0,550 \\
\hline $2^{\circ}$ trimestre & 16 & 43,2 & 6 & 26,0 & 5 & 50,0 & \\
\hline $3^{\circ}$ trimestre & 6 & 16,2 & 12 & 52,1 & 2 & 20,0 & \\
\hline
\end{tabular}


(Conclusão)

\begin{tabular}{|c|c|c|c|c|c|c|c|}
\hline \multirow{3}{*}{ Variáveis } & \multicolumn{6}{|c|}{ Ganho ponderal total } & \multirow{3}{*}{ Valor $\mathrm{p}$} \\
\hline & \multicolumn{2}{|c|}{$\begin{array}{l}\text { Insuficiente } \\
\quad(n=37)\end{array}$} & \multicolumn{2}{|c|}{$\begin{array}{c}\text { Adequado } \\
(n=23)\end{array}$} & \multicolumn{2}{|c|}{$\begin{array}{c}\text { Excessivo } \\
(n=10)\end{array}$} & \\
\hline & $\mathrm{n}$ & $\%$ & $\mathrm{n}$ & $\%$ & $\mathrm{n}$ & $\%$ & \\
\hline Situação marital & & & & & & & 0,022 \\
\hline Sem companheiro & 16 & 43,2 & 10 & 43,5 & 0 & 0,0 & \\
\hline Com companheiro & 21 & 56,8 & 13 & 56,5 & 10 & 100,0 & \\
\hline Escolaridade & & & & & & & 0,414 \\
\hline$<8$ anos & 21 & 56,8 & 9 & 39,1 & 5 & 50,0 & \\
\hline$\geq 8$ anos & 16 & 43,2 & 14 & 60,9 & 5 & 50,0 & \\
\hline Ocupação & & & & & & & 0,998 \\
\hline Não remunerada & 26 & 70,3 & 16 & 69,3 & 7 & 70,0 & \\
\hline Remunerada & 11 & 29,7 & 7 & 30,4 & 3 & 30,0 & \\
\hline Renda familiar* & & & & & & & 0,427 \\
\hline$\leq 1$ salário & 23 & 62,2 & 12 & 52,2 & 4 & 40,0 & \\
\hline$>1$ salário & 14 & 37,8 & 11 & 47,8 & 6 & 60,0 & \\
\hline Estado Nutricional Gestacional & & & & & & & 0,427 \\
\hline Baixo peso & 8 & 21,6 & 4 & 17,3 & 4 & 40,0 & \\
\hline Eutrofia & 12 & 32,4 & 11 & 47,8 & 3 & 30,0 & \\
\hline Sobrepeso & 12 & 32,4 & 7 & 30,4 & 1 & 10,0 & \\
\hline Obesidade & 5 & 13,5 & 1 & 4,3 & 2 & 20,0 & \\
\hline
\end{tabular}

As gestantes classificadas com sobrepeso apresentaram média de idade de 27,9 $( \pm 7,1)$ anos superior à observada nos demais grupos. Ainda, observou-se que o grupo de gestantes com sobrepeso apresentava-se com média de idade gestacional superior aos demais grupos. Os dados evidenciaram a associação significativa entre o IMC pré-gestacional e o estado nutricional $(p<0,000)$. Dessa forma, as mães que foram diagnosticadas com baixo peso e obesidade durante a gestação apresentavam IMC pré-gestacional médio de 18,6 e 34,5, respectivamente (Tabela 3).

No que diz respeito ao consumo alimentar, não foi possível notar diferença nas médias da quantidade diária de energia, nos diferentes grupos classificados pelo IMC. Quanto à proporção de distribuição dos macronutrientes, não foi verificada diferença significativa entre os grupos analisados. A ingestão diária de ferro, cálcio, vitamina $\mathrm{C}$, zinco e ácido fólico foi semelhante entre os grupos. Quanto à ingestão de vitamina $\mathrm{A}$, a quantidade ingerida, por dia, foi maior nas gestantes obesas quando comparadas às com baixo peso, eutrofia $\mathrm{e}$ sobrepeso. Na avaliação da ingestão de fibras, observou-se que os resultados indicam menor ingestão nas gestantes com excesso de peso quando comparadas às eutróficas, sendo essa diferença não significativa (Tabela 3). 
Tabela 3. Características maternas e consumo dietético segundo o estado nutricional de gestantes atendidas em Estratégias de Saúde da Família, Alfenas-MG, 2017

\begin{tabular}{|c|c|c|c|c|c|c|c|c|c|c|}
\hline \multicolumn{2}{|c|}{$\begin{array}{c}\text { Características maternas/consumo } \\
\text { energético }\end{array}$} & \multicolumn{2}{|c|}{$\begin{array}{l}\text { Baixo peso } \\
\quad(n=16)\end{array}$} & \multicolumn{2}{|c|}{ Eutrofia $(n=26)$} & \multicolumn{2}{|c|}{$\begin{array}{l}\text { Sobrepeso } \\
(n=20)\end{array}$} & \multicolumn{2}{|c|}{ Obesidade $(\mathrm{n}=8)$} & \multirow[t]{2}{*}{ Valor $\mathbf{p}$} \\
\hline & & Média & DP & Média & DP & Média & DP & Média & DP & \\
\hline \multicolumn{2}{|c|}{ Idade materna (anos) } & 27,1 & 6,4 & 23,8 & 3,9 & 27,9 & 7,1 & 26,3 & 6,0 & 0,182 \\
\hline \multicolumn{2}{|c|}{ IMC pré-gestacional $\left(\mathrm{kg} / \mathrm{m}^{2}\right)$} & 18,6 & 1,5 & 22,3 & 1,6 & 27,9 & 1,3 & 34,5 & 3,3 & 0,000 \\
\hline \multicolumn{2}{|l|}{ IG (semanas) } & 20,1 & 10,7 & 16,9 & 10,1 & 22,3 & 10,4 & 20,7 & 9,8 & 0,317 \\
\hline \multicolumn{2}{|l|}{ Energia (kcal) } & 1492,1 & 544,3 & 1431,6 & 391,0 & 1191,9 & 496,8 & 1244,8 & 556,1 & 0,204 \\
\hline \multicolumn{11}{|c|}{ Macronutrientes (g) } \\
\hline \multicolumn{2}{|l|}{$\mathrm{CHO}$} & 190,7 & 81,4 & 190,7 & 70,1 & 145,0 & 63,2 & 148,0 & 67,3 & 0,090 \\
\hline \multicolumn{2}{|l|}{ PTN } & 69,9 & 31,7 & 68,7 & 24,6 & 65,9 & 35,6 & 70,13 & 40,3 & 0,984 \\
\hline \multicolumn{2}{|l|}{ LIP } & 49,1 & 24,4 & 43,8 & 19,1 & 38,7 & 23,3 & 41,3 & 19,1 & 0,573 \\
\hline Micronutrientes & $\begin{array}{l}\text { Necessidade } \\
\text { diária }\end{array}$ & & & & & & & & & \\
\hline FIB (g) & 28 (AI) & 12,7 & 5,6 & 16,15 & 8,7 & 11,9 & 5,3 & 13,2 & 4,8 & 0,182 \\
\hline Ferro (mg) & 27 (RDA) & 11,3 & 3,8 & 11,35 & 3,4 & 9,9 & 4,2 & 10,2 & 5,6 & 0,609 \\
\hline Cálcio (mg) & 1000 (AI) & 437,9 & 237,7 & 463,1 & 275,9 & 327,9 & 219,7 & 328,2 & 279,6 & 0,480 \\
\hline VIT. A (mcg) & 550 (EAR) & 300,3 & 335,2 & 503,4 & 631,9 & 637,0 & 1803,7 & 1348,1 & 2830,4 & 0,194 \\
\hline VIT.C (mg) & 70 (EAR) & 53,6 & 93,0 & 93,9 & 97,5 & 43,0 & 39,0 & 54,5 & 73,6 & 0,339 \\
\hline Folato (mcg) & 520 (EAR) & 147,8 & 94,8 & 148,3 & 75,9 & 126,2 & 64,3 & 135,5 & 83,6 & 0,872 \\
\hline Zinco (mg) & 9,5 (EAR) & 7,3 & 4,8 & 7,3 & 4,3 & 8,0 & 5,5 & 6,5 & 4,8 & 0,840 \\
\hline
\end{tabular}

Fontes: IOM, 1990, 1997, 2002, 2005. Nota: IMC: Índice de Massa Corporal; DV: Desvio Padrão. AI: Adequate Intake; RDA: Recommended Dietary Allowances; EAR: Estimated Average Requirement

\section{DISCUSSÃO}

Este estudo teve como objetivo avaliar a atenção nutricional ofertada ao pré-natal pela rede básica do Sistema Único Saúde (SUS) do município de Alfenas, segundo análise do estado nutricional, envolvendo avaliação antropométrica e consumo alimentar de gestantes, bem como o conhecimento do ganho ponderal materno. Um dos componentes essenciais para a integralidade da atenção ao pré-natal é a atenção nutricional, que compreende cuidados de alimentação e nutrição voltados à promoção e à proteção da saúde, prevenção, diagnóstico e tratamento de agravos 9 . O conhecimento do consumo dietético e os extremos do estado nutricional aprimora a qualidade do atendimento, e pode ser essencial para fortalecer o planejamento e desenvolvimento saudável da gravidez, minimizando riscos de comprometimento materno e perinatal ${ }^{2}$.

Neste estudo, os resultados evidenciaram a prevalência de gestantes inseridas na faixa etária entre 20 a 30 anos, dado que foi similar ao verificado em outra pesquisa realizada em Juiz de Fora-MG, que constatou a prevalência em torno 52\% de idade materna entre 20 a 30 anos $^{14}$. Tal período é tido como ótimo do ponto de vista reprodutivo, uma vez que de acordo com dados recentes da literatura, a ocorrência de parto prematuro e morbimortalidade perinatal é superior em adolescentes e também em mulheres que possuem mais de 30 anos $^{15}$.

No que se refere às variáveis socioeconômicas, notou-se que metade das gestantes possuíam menos de oito anos de estudo, além de maior prevalência de mulheres casadas ou com união estável e sem ocupação 
com remuneração e que sobrevivem com menos de um salário mínimo; indicadores parecidos foram encontrados pelos autores Santos et al. ${ }^{16}$ Esses indicadores já eram esperados, uma vez que tradicionalmente a ESF se baseia em uma política de saúde equitativa, sendo prioritariamente alocada nos bairros mais carentes dos municípios, a fim de favorecer aos mais pobres e reduzir as diferenças socioeconômicas ao acesso a serviços básicos de saúde ${ }^{17}$.

Os dados supracitados constituem fatores de risco para desfechos perinatais ruins, ainda, repercutem negativamente no consumo de alguns alimentos em detrimento de outros, nas condições de vida e saúde, já que baixa escolaridade leva maior dificuldade em compreender os cuidados com a saúde da gestante acarretando início tardio do pré-natal ou ausência dele, alimentação e hábitos de vida inadequados ${ }^{7}$. Por outro lado, a presença paterna favorece o desenvolvimento de uma gestação confortável, por transmitir segurança e divisão e/ou compartilha das dificuldades e das responsabilidades desta fase ${ }^{18}$.

Observou-se que das gestantes entrevistadas 8,6\% e 11,4\% admitiram consumir durante a gravidez álcool e tabaco, respectivamente. Tal percentual foi inferior ao verificado em estudos realizados no interior do Rio Grande do Sul e em uma maternidade do sul de Santa Catarina, segundo os quais, $48,1 \%$ e $36,9 \%$ das grávidas fazem uso de álcool e 44,6\% e 14\% assumiram fumar durante a gravidez, respectivamente ${ }^{19-20}$. Entretanto, a existência de mulheres que consomem álcool e tabaco durante a gravidez, ainda que em baixo percentual de acordo com os dados encontrados nesta pesquisa, evidencia a necessidade de maior discussão com a comunidade sobre a importância da abstinência de álcool e tabaco durante o período gestacional, uma vez que o consumo de drogas na gestação traz implicações ao feto que sofre os impactos do álcool e das múltiplas substâncias do tabaco, que é o grande causador de abortos, ruptura de membrana e descolamento de placenta ${ }^{20}$.

No presente estudo foi possível observar que o número de mulheres com baixo peso aumentou no decorrer do período gestacional quando comparado com o estado pré-gestacional, já as gestantes que apresentaram sobrepeso e obesidade se mantiveram com esse diagnóstico, dado semelhante encontrado pelos autores Pereira e Wichmann ${ }^{21}$, na pesquisa conduzida com gestantes cadastradas em uma Unidade Básica de Saúde. Dentre as hipóteses para os resultados mencionados pode-se citar o baixo poder aquisitivo, o qual limita a compra de gêneros alimentícios, e também a presença de náusea e vômitos, que são tidos como sintomas comuns durante a gravidez, afetando de $50 \%$ a $90 \%$ das gestantes, podendo cursar com hiporexia e ingestão alimentar insuficiente ${ }^{4}$

Assim como na pesquisa de Fazio et al. ${ }^{2}$, nesse levantamento, também foi verificada associação significativa entre o IMC pré-gestacional e o estado nutricional durante a gestação. A prevalência de ganho ponderal excessivo foi de $14,2 \%$, valor bem inferior ao encontrado em estudo realizado em Jundiaí-SP $(36,9 \%)^{22}$.

No presente estudo, as gestantes que possuíam companheiro apresentaram prevalência significativamente maior de ganho de peso acima do recomendado, quando comparadas com as mulheres solteiras. A associação positiva entre ganho excessivo de peso e a presença de companheiro pode ser explicada pelo contexto de maior apoio familiar que tais mulheres recebem o que pode ser um fator favorável ao consumo energético excessivo. Assim, justifica-se o monitoramento do ganho de peso, já que o ganho ponderal em excesso e a obesidade na gravidez estão intimamente relacionados com maiores riscos adversos para a mãe e recémnascido no pós-parto, além de promover o aumento dos marcadores inflamatórios durante a gestação, devendo ser evitado ${ }^{6,23}$.

Por outro lado, o ganho de peso insuficiente foi registrado em mais de metade das gestantes, índice também preocupante, visto que o ganho ponderal gestacional abaixo do recomendado está ligado ao baixo-peso ao nascer e ao nascimento pré-termo ${ }^{24}$. A determinação do estado nutricional durante a gestação é parte fundamental na caracterização dos aspectos da saúde materno-infantil de regiões e municípios ${ }^{21}$. Assim, é permitida a elaboração de políticas de prevenção e programas de saúde mais eficazes e direcionados a atender as reais necessidades das gestantes.

Conhecer a ingestão de nutrientes das gestantes é de extrema importância para fundamentar novas 
intervenções alimentares durante esse período da vida, sendo assim, subsídios para o desenvolvimento saudável da gestação. A partir do Recordatório Alimentar de 24 horas, foi possível estimar que o consumo de carboidratos na proporção de 48 a 53\%, da ingestão calórica total, apresentou pequena variação entre os estados nutricionais. O resultado encontrado pode ser considerado adequado segundo as recomendações para gestantes (AMDR) e percentuais semelhantes também foram encontrados em estudo realizado em Juiz de Fora $-\mathrm{MG}(55 \%)^{14}$.

O consumo proteico médio verificado foi de 68 gramas ao dia $(1,06 \mathrm{~g} / \mathrm{kg} / \mathrm{dia})$, valor dentro do recomendado (10 - 35\% do valor energético total) $(\mathrm{AMDR})^{13}$. Em um levantamento realizado na Dinamarca, as gestantes consumiam em média 78 gramas de proteínas ao dia; a diferença entre o consumo proteico das gestantes dos dois estudos citados pode ser explicada pelas diferenças socioeconômicas entre as populações e em virtude de distinções culturais e hábitos alimentares dos dois países ${ }^{25}$. As proteínas são responsáveis por funções essenciais durante o período gestacional, tais como formação de tecidos fetais e anexos e a deficiência da mesma associa-se ao retardo de crescimento intrauterino e desenvolvimento de pré-eclâmpsia ${ }^{1}$.

No que se refere à média do consumo de lipídios, a mesma se mostrou dentro das recomendações de 20 a $35 \%$ (AMDR) em todos os estados nutricionais ${ }^{13}$. Embora a proporção de consumo de gorduras esteja adequada, há a preocupação quanto à qualidade das mesmas, uma vez que é de comum senso que a alimentação típica do brasileiro é rica em gorduras saturadas e trans, as quais afetam diretamente a saúde do sistema cardiovascular e contribuem para o ganho ponderal excessivo.

A investigação em questão revelou que as gestantes pesquisadas apresentam prevalência de inadequação de todos os micronutrientes analisados, quando comparadas com as recomendações adotadas, exceto vitamina A. A adequação do consumo de vitamina A pode ser esclarecida pelo amplo consumo e fácil acesso de alimentos fontes dessa vitamina no Brasil, outros levantamentos realizados no país encontraram dados parecidos $^{2,7,14}$.

A inadequação na ingestão de fibras, ferro, cálcio, vitamina $\mathrm{C}$, folato e zinco pode ser resultante da baixa renda familiar da população estudada, visto que uma meta-análise da ingestão de micronutrientes durante a gravidez em países desenvolvidos encontrou consumo acima da Estimated Average Requerement (EAR) para micronutrientes e minerais como vitamina A, vitamina C, cálcio e zinco, dentre os nutrientes pesquisados em tal estudo, foram encontradas inadequações apenas para ingestão de folato, ferro e vitamina $\mathrm{D}$, que são nutrientes comumente suplementados durante a gestação em virtude da dificuldade em atingir os requerimentos recomendados ${ }^{26-27}$.

O presente trabalho teve como limitações as características socioeconômicas homogêneas da população e não permitem explorar os resultados para outras populações. Outra limitação verificada foi o pequeno número de gestantes captadas durante o estudo. Além disso, o uso do Recordatório Alimentar de 24 horas na mensuração do consumo alimentar, uma vez que se refere apenas um dia, no entanto, o mesmo é largamente usado em pesquisas por se tratar de um método prático, rápido e de baixo custo.

\section{CONCLUSÃO}

Os resultados deste estudo permitem concluir que houve prevalência de ganho de peso insuficiente e a qualidade da alimentação da população avaliada mostrouse aquém das recomendações preconizadas, sendo assim, apontam para a necessidade da criação de estratégias que trabalhem melhor a segurança alimentar e nutricional das futuras mães e a importância da alimentação adequada para que, consequentemente, melhorem o desfecho da gestação. Ainda, os dados indicam a necessidade imperiosa de melhorar a qualidade da atenção nutricional no município pesquisado, incluindo o cuidado multiprofissional, na rede primária de saúde.

\section{REFERÊNCIAS}

1. Vitolo, MR. Nutrição: da gestação ao envelhecimento. 2. ed. Rio de Janeiro: Rubio; 2015.

2. Fazio EDS, Nomura RMY, Dias MCG, Zugaib M. Consumo dietético de gestantes e ganho ponderal materno após aconselhamento nutricional. Rev. bras . ginecol. Obstet. 2011;33(2):87-92. 
3. Brasil. Ministério da Saúde. Atenção ao pré-natal de baixo risco. Secretaria de Atenção à Saúde. Departamento de Atenção Básica. Brasília (DF): Ministério da Saúde; 2012.

4. Fernandes CE. Medicamentos antieméticos no tratamento da náusea e vômitos associados à gestação. RevBras Med. 2013; 70(6),227-31.

5. Imdad A,Bhutta ZA. Nutritional management of the low birth weight/preterm infant in community settings: a perspective from thedeveloping world. J. Pediatr. 2013;162(3):S107-S114.

6. Magalhães EIS, Maia DS, Bonfim CFA, Netto MP, Lamounier JÁ, Rochaii DS. Prevalência e fatores associados ao ganho de peso gestacional excessivo em unidades de saúde do sudoeste da Bahia. Rev. bras. epidemiol. 2015;18(4):858-69.

7. Lacerda SKS, Macedo KGF, Freire JAP, Voci SM. Prevalência da inadequação no consumo de nutrientes entre gestantes atendidas em unidades básicas de saúde. Rev. bras. educ. méd. 2014;27(3).

8. World Health Organization (WHO). Global database on body mass index: an interactive surveillance tool for monitoring nutrition transition. WHO; 2012.

9. Laporte-Pinfildi ASDC, Zangirolani LTO,Spina N,Martins PA, Medeiros MAT. D. Atenção nutricional no pré-natal e no puerpério: percepção dos gestores da Atenção Básica à Saúde. Rev. nutr. 2016;29(1): 109-23.

10. World Health Organization (WHO). Global standards for quality health-care services for adolescents: A guide to implement a standards-driven approach to improve the quality of health-care services for adolescents. WHO; 2015.

11. Brasil. Ministério da Saúde. Orientações para coleta e análise de dados antropométricos em serviços de saúde: norma técnica do sistema de Vigilância Alimentar e Nutricional - SISVAN. Brasília (DF): Ministério da Saúde; 2011.

12. Institute Of Medicine (IOM). Weight gain during the pregnancy: Reexamining the Guidelines. Washington
(DC): National Academy Press; 2009.

13. Institute Of Medicine (IOM). Dietary Reference Intakes for Vitamin A, Vitamin K, Arsenic, Boron, Chromium, Copper, Iodine, Iron, Manganese, Molybdenum, Nickel, Silicon, Vanadium, and Zinc. Washington (DC): National Academy Press; 2002.

14. Institute Of Medicine (IOM). Dietary Reference Intakes for Calcium, phosphorus, magnesium, vitamin D, and fluoride. Washington (DC): National Academy Press; 1997.

15. Institute Of Medicine (IOM). Dietary reference intakes for energy, carbohydrate, fiber, fat, fatty acids, cholesterol, protein, and amino acids. Washington (DC): NationalAcademy Press; 2005.

16. Institute Of Medicine (IOM). Subcommittee on Nutritional Status and Weight Gain During Pregnancy. Nutrition during pregnancy: part I, weight gain; part II, nutrient supplements. Washington (DC): NationalAcademy Press; 1990.

17. Andrade BD, Silva ACP, Santos MTMD, Campos T, Luquetti SCPD, Cândido APC, et al. Fatores Nutricionais e Sociais de Importância para o Resultado da Gestação, em Mulheres em Acompanhamento na Rede de Atenção Primária de Juiz de Fora. Juiz de Fora. Rev. méd. Minas Gerais. 2015;25(3):344-52.

18. Lisonkova S, Potts J, Muraca GM, Razaz N, Sabr Y, Chan WS, et al. Maternal age and severe maternal morbidity: A population-based retrospective cohort study. PloS med. 2017;14(5):e1002307.

19. Santos JGC, Silva JMC, Passos AMPR, Monteiro BKSM, Maia MM, Silva RA, Santos J. et al. Peso materno em gestantes de baixo risco na atenção pré-natal. Int J. Nutrology. 2017;10(2):5-15.

20. Andrade MV, Noronha K, Barbosa ACQ, Rocha TAH, Da Silva NC, Calazans JÁ, et al. A equidade na cobertura da Estratégia Saúde da Família em Minas Gerais, Brasil. Cad. saúde pública. 2015;31(6):117587. 
21. Araújo ES, Santana JM, Brito SM, Santos DB. Consumo alimentar de gestantes atendidas em Unidades de Saúde. Mundo Saúde. 2016;40(1):28-37.

22. Maria FN, Jornada LK, Sakae TM, Cassol Jr OJ, Sakae DY, Quevedo JL. Uso de álcool e tabaco por gestantes em maternidade do sul de Santa Catarina. ACM arq. catarin. med.2016;44(1):41-61.

23. Renner FW, Costa BP, Figueira FP, Ebert JP, Nascimento LS, Ferrari L, et al. Avaliação do uso de drogas por gestantes atendidas em hospital de ensino do interior do Rio Grande do Sul. Rev. epidemiol. controle infecç. 2016;6(2):68-73.

24. Pereira VR, Wichmann FMA. Estado nutricional materno e peso ao nascer do bebê no município de Candelária - RS. Cinergis. 2016;17(Supl.1): 368-72.

25. Fonseca MRCC, Laurenti R, Roveri Marin C, Traldi MC. Ganho de peso gestacional e peso ao nascer do concepto: estudo transversal na região de Jundiaí, São Paulo, Brasil. Ciênc. Saúde Colet. 2014;19(5): 1401-07.

26. Nascimento IB, Dienstmann G, De Souza MLR, Fleig R, Silva JC. Os Efeitos da Metformina na Obesidade da Gestante e no Peso do Recém-Nascido. Saude e pesqui. 2017;10(2):373-82.

27. Haugen M, Brantsæter AL, Winkvist A, Lissner L, Alexander J, Oftedal B, et al. Associations of prepregnancy body mass index and gestational weight gain with pregnancy outcome and postpartum weight retention: a prospective observational cohort study. BMC pregnancy childbirth. 2014;14(1):201.

28. Hrolfsdottir L, Schalkwijk CG, Birgisdottir BE, Gunnarsdottir I, Maslova E, Granström C. Maternal diet, gestational weight gain, and inflammatory markers during pregnancy. Obesity. 2016;24(10): 2133-39.

29. Basile LH. Gestante e necessidade da vitamina D. Int J. Nutrology. 2014;7(1):5-13.

30. Blumfield ML, Hure AJ, Macdonald-Wicks L, Smith R,
Collins CE. A systematic review and meta-analysis of micronutrient intakes during pregnancy in developed countries. Nutr. rev. 2013;71(2):118-32.

Recebido em: 05/01/2018

Aceito em: 25/05/2018 